\title{
Klinische Ethik neu denken
}

\author{
Medizinische Ethik ist mehr als nur Entscheidungsfindung. Jeder Mitarbeiter muss \\ langfristig befähigt werden, seine Handlungen ethisch zu reflektieren. Ethik wird so \\ zur Bildungsaufgabe.
}

\section{Rouven Porz ${ }^{a}$, \\ Kurt Laederach ${ }^{b}$}

a Rouven Porz ist Leiter der Ethikstelle im Inselspital Bern, Gastwissenschaftler am Institut für Biomedizinische Ethik in Zürich und Redaktionsmitglied der SÄZ.

b Kurt Laederach ist der Vorsitzende der hausinternen Ethikkommission des Inselspitals Bern, Leitender Arzt an der Poliklinik für Endokrinologie, Diabetologie und Klinische Ernährung und Forschungsgruppenleiter im Bereich Ernährungspsychologie und Adipositas, Inselspital Bern.
Interessenkonflikt:

Die dargestellten Meinungen entsprechen denen der Autoren und müssen nicht notwendigerweise mit den Meinungen der Mitarbeitenden der beteiligten Institutionen übereinstimmen.

Korrespondenz:

Dr. phil. dipl. biol. Rouven Porz Ethikstelle Inselspital

Universitätsspital Bern Murtenstrasse 10

CH-3010 Bern

rouven.porz@insel.ch
In unseren westlichen Gesellschaftsformen, und damit auch in unseren Gesundheitssystemen, lässt sich zunehmend ein immer lauter werdender Ruf nach Ethik vernehmen. In Spitälern zeigt sich dieser Trend zum Beispiel darin, dass eine Vielzahl von Ethikstrukturen institutionalisiert angeboten bzw. entwickelt werden: Ethikkommissionen, Ethikkomitees, Ethikforen, Ethikberatung, Ethikbeauftragte, Ethikstellen wir als Autoren dieses Textes sind Teil dieser Entwicklung, Teil der sogenannten «klinischen Ethik», unter anderem als Vorsitzender einer Ethikkommission und als Leiter einer Ethikstelle in einem Universitätsspital. Dennoch betrachten wir diese «Inflation» der Ethik mit einer gewissen selbstkritischen Vorsicht. Dies nicht, weil wir nicht von dem Grundgedanken einer humanistischen Ethik überzeugt wären, ganz im Gegenteil, aber wir finden, dass die Institutionalisierung von Ethik auch Gefahren der Vereinfachung mit sich bringen kann. Wir wollen vorliegend zwei solche von uns wahrgenommenen Vereinfachungen näher betrachten: Das Verständnis von Ethik als Disziplin der Entscheidungsfindung in heiklen klinischen Situationen sowie das medizinethische Primat der Patientenautonomie. Wir bitten die Leserinnen und Leser, eventuelle Vereinfachungen von unserer Seite ebenso kritisch zu betrachten, wie wir es mit unserer eigenen Disziplin der Ethik versuchen.

\section{Ethik als Entscheidungsfindung?}

Um es direkt und deutlich zu sagen: Aus unserer Sicht ist Ethik mehr als nur Entscheidungsfindung. Ethik beschäftigt sich mit menschlichen Handlungen. Im Gesundheitssystem laufen vielschichtige Handlungsketten ab. Jede einzelne Mitarbeiterin - z. B. in einem Spital - muss langfristig befähigt sein, diese Handlungen ethisch zu reflektieren, und sie muss sich über den eigenen moralischen Horizont bewusst sein. Somit ist die institutionalisierte klinische Ethik - in unserer Sichtweise - vor allem eine Bildungsaufgabe. Dennoch kam (und kommt) der institutionalisierten Ethik bislang vor allem in der sogenannten «Ethikberatung» oftmals nur die Rolle zu, als (weitere) Disziplin in der medizinischen Entscheidungssuche helfend und orientierend wirken zu müssen, im besten Fall, indem «Ethik-Experten» mithelfen, Wertekonflikte zu entschlüsseln und sinnvolle Handlungsoptionen in einer Dilemma-Situation anzubieten. Hierzu gibt es auch

\section{Repenser l'éthique clinique}

L'éthique traite de la morale et des valeurs humaines. Ces dernières années, elle a fait son entrée dans les hôpitaux et institutions de notre système de santé par le biais d'une sous-discipline appelée éthique clinique. Or cette discipline court le risque de se faire du tort à long terme si, dans le monde clinique, elle se définit uniquement comme étant centrée sur les prises de décision ou si elle ne parvient pas à rendre compréhensible, dans le quotidien hospitalier, des idéaux humanistes tels que l'autonomie du patient. Par notre travail, nous essayons de contrer ces simplifications, car pour nous, l'éthique s'entend principalement comme une activité formatrice. L'article ci-après présente quelques réflexions conceptuelles à ce sujet.

eine Vielzahl von Entscheidungsfindungsmodellen, die diesen schwierigen Prozess der heiklen klinischen Entscheidungsfindung in Einzelschritte zu unterteilen versuchen, Verantwortungen verteilen und TeamEntscheidungen stärken (obwohl - oder gerade weil die rechtliche Verpflichtung letztendlich doch immer beim behandelnden Facharzt liegt). Gerade bei uns in der Schweiz scheint auch der Moment der Konsensfindung im Entscheidungsprozess durchaus wichtig zu sein, obwohl eine im Konsens getroffene Entscheidung aus logischer Sicht noch keine gute Entscheidung sein muss.

Aber warum skizzieren wir diese Entwicklung der klinischen Ethik als eine Vereinfachung? Wir sprechen deshalb von einer Vereinfachung, weil es in diesem Prozess der Institutionalisierung von Ethik epistemologische Gefahren gibt, die der Disziplin langfristig schaden könnten. Eine solche Gefahr (bzw. ein möglicher langfristiger Schaden) liegt in den falschen Erwartungen gegenüber der Disziplin der Ethik, die durch das Versprechen einer ethischen Entscheidungsfindung ausgelöst werden. Die Ethik kann keine Para- 
delösungen bieten. Generell sind die heiklen klinischen Entscheidungssituationen - von denen wir hier sprechen - meistens so komplex, dass es auch oftmals gar keine gute Lösung geben kann. Salopp formuliert: Für mindestens einen der Beteiligten geht eine Dilemma-Situation in der eigenen Befindlichkeit oftmals nicht gut aus, sei es für den Patienten, die Angehörigen, den Arzt oder die Pflegenden. Dieser Umstand muss deutlich ausgesprochen werden und darf nicht durch eine scheinbare «ethische» Entscheidungsfindung heruntergespielt werden. Klinisch-komplexe Situationen sind oftmals tragisch, existenziell herausfordernd oder flektieren. Der Spitalalltag ist voll von Handlungen bzw. Handlungsketten. Die meisten dieser Handlungen laufen routiniert und unbewusst ab, nur manchmal kommen Handlungen zum Stocken, gerade in heiklen Dilemma-Situationen, und dann wird der Ruf nach Ethik laut. Eine aufgeklärte klinische Ethik [1] sollte sich möglichst die gesamten Handlungsketten anschauen, organisationsethisch und strategisch denken und nicht nur den Moment betrachten, in dem eine Einzelhandlung als Dilemma aufbricht. Dies kann z.B. dadurch geschehen, dass die moralisch-ethischen Kompetenzen der Mitarbeitenden langfristig geschult

\section{Ethik muss Menschen ermutigen und befähigen, über ihre Wertvorstel- lungen zu sprechen und ihre Handlungen selbstkritisch zu reflektieren}

einfach sehr bedauerlich. Aber damit sind sie auch kaum in einem einfachen Entscheidungsfindungsmodell abzubilden, dies ist eine Vereinfachung, die langfristig dazu führen kann, dass der Disziplin der Ethik ihre Glaubhaftigkeit abgesprochen wird, weil die beteiligten Mediziner und Pflegenden merken werden, dass entweder immer wieder ein schlechtes Bauchgefühl zurückbleibt, oder dass trotz interdisziplinärer Entscheidungsfindung dennoch letztendlich der Chefarzt entscheidet, oder dass die unvermeidbare existenzielle Unsicherheit einer Dilemmasituation durch eine scheinbare Verantwortungsteilung aufzulösen versucht wird. Die Ethik kann ein wirkliches Dilemma niemals auflösen, sie kann es höchstens artikulierbar und damit verstehbar machen. Darüber hinaus sind die besprochenen «ethischen» Probleme oftmals juristischer Natur, und die Angst vor einer möglichen Strafverfolgung kann die «ethische Entscheidung» als moralische Absegnung erscheinen lassen. Es gilt auch zu beachten, dass die Ethik nicht als medizinischer Konsiliardienst wirken kann, denn die Ethik ist in Argumentation und Begründung keine naturwissenschaftliche bzw. medizinische Disziplin (obwohl auch die klassische Medizin, historisch gesprochen, eher eine hermeneutische Handlungskunst ist und keine reine Naturwissenschaft).

Warum versuchen wir nicht, die Institutionalisierung von Ethik neu zu denken? Die klinische Ethik handelt nicht nur von Entscheidungsfindungen, sondern von menschlichen Handlungen im Gesundheitswesen. Sie beschäftigt sich mit Wert- und Moralvorstellungen von (oftmals sehr engagierten) Menschen in Berufsrollen. Oft ist es aber noch nicht einmal diesen einzelnen Beteiligten klar, welchen Werten sie sich selbst verpflichtet fühlen, und wie sie ihre eigenen Moralvorstellungen in Worte fassen sollen. Die Ethik muss genau dort ansetzen. Sie muss Menschen ermutigen und befähigen über ihre Wertvorstellungen zu sprechen und ihre Handlungen selbstkritisch zu re- werden, ganz einfach z. B. durch fest implementierte hausinterne Weiterbildungen und regelmässige ethische Fallbesprechungen. Die Ärztin oder der Pflegende müssen selbst, als Personen, langfristig dazu befähigt werden, eigene Wertkonflikte benennen, bzw. diese im Team artikulieren zu können. Sie müssen in der Lage sein, die eigenen Moralvorstellungen zu reflektieren und diese in Relation zu den Werten ihrer Berufsrolle zu setzen. Damit ist die klinische Ethik keine reine Beratungsdisziplin mehr, sondern eher eine Bildungsaufgabe, die sich primär mit den Health Care Professionals selbst beschäftigen sollte.

Die Ethik gehört somit in die Curricula der Weiterbildung, ins Studium von Pflegenden und Medizinern, und sie muss analytische und konzeptuelle Werkzeuge liefern, um menschliche Handlungsketten verstehbar und artikulierbar zu machen. In einem zweiten Schritt wird sie dann notwendigerweise auch den Patienten zugutekommen, schliesslich werden diese von den Behandlungsteams - und nicht von Ethik-Experten - betreut.

\section{Patientenautonomie?}

Wenn heutzutage in unserem Gesundheitssystem von Patienten die Rede ist, dann oft im selben Atemzug mit der Betonung ihrer Autonomie. Obwohl man sich im Alltag kaum darüber verständigt, was mit dieser Autonomie überhaupt gemeint sein könnte, so lässt sich nicht mehr daran zweifeln, dass Patienten generell eine Autonomiefähigkeit zugesprochen wird. Dies ist mit Sicherheit gut so, zumindest so lange, wie Patienten diese Fähigkeit ausleben können; also solange sie frei und unvoreingenommen, wohl informiert möglichen Diagnosen und Therapien zustimmen - oder diese ablehnen - können. Ethisch wird es natürlich immer dann brisant, wenn Unmündige, Kinder, Koma-Patienten, Psychisch-Kranke, oder z.B. sehr kranke oder sehr alte Menschen ihre Autonomie nicht ausleben können, wenn Autonomieanspruch 
und Autonomiefähigkeit nicht mehr zu vereinen sind. Dabei entsteht möglicherweise beim Behandlungsteam der Eindruck, dass sie bei diesen Patienten per se deren Autonomie verletzen, weil sie diese nicht mehr abfragen können. Wir glauben, dass hier eine Gefahr, ja ein Missverständnis vorliegt, an dem die Vertreter der Medizinethik nicht ganz unschuldig sind. Denn gerade im Unterricht von Medizinethik wird die Patientenautonomie oft als intrinsischer Faktor des Patienten dargestellt. Provokativ könnte man sagen: Ein moderner Patient muss heutzutage von seiner Autonomie Gebrauch machen, um sozusagen compliant zu sein, und wenn er oder sie das nicht mehr kann, dann scheint es ethisch schwierig zu werden. Das ist aber eine zu vereinfachte Sichtweise des Ideals der Patientenautonomie.

Wenn wir nämlich die «Patientenautonomie» als konzeptuelles ethisches Ideal betrachten und nicht als operationalisierbare und abrufbare Handlungsweise des Patienten, dann wird zumindest aus theoretischethischer Sicht deutlich, dass es im Alltag von Medizinern und Pflegenden nicht nur darum gehen kann, die «Patientenautonomie» abzurufen, sondern vielmehr darum, sich in der eigenen Berufsrolle immer wieder des «Respekts» gegenüber der Patientenautonomie bewusst zu sein. Die in der Medizinethik viel zitierten Vertreter der sogenannten vier biomedizinethischen Grundprinzipien (Tom Beauchamp und James Childress) führen dies in ihrem Standardwerk Principles of Biomedical Ethics zwar sehr deutlich aus, leider wird aber gerade das erste und prominenteste Prinzip, der «Respekt vor der Patientenautonomie», oftmals im klinischen Alltag und im Gesundheitssys-

\section{Man kann sich auch gegenüber einem nicht autonomiefähigen Patienten respektvoll verhalten}

tem vereinfacht nur als «Patientenautonomie» zitiert und wahrgenommen. Diese Präzisierung ist weitaus mehr als nur eine Nuance oder eine begriffliche Erweiterung. Es ist eine Änderung in der Grundeinstellung gegenüber den Patienten [2]. Ich kann mich nämlich auch gegenüber einem nicht autonomiefähigen Patienten respektvoll verhalten, ohne mich als behandelnde Pflegende oder Ärztin ständig moralisch fragwürdig zu fühlen, wenn es mir nicht permanent gelingt, dessen Autonomie abzurufen. Dies ist auch keine erneute Türöffnung für den ärztlichen Paternalismus oder gar ein Rückschritt in diesen, es ist lediglich eine berufsethische Präzisierung: In der Berufsrolle eines Health Care Professionals in unserer westlichen Welt ist das oberste Primat der Respekt vor der Patientenautonomie, nicht die Autonomie selbst. Wenn also ein Patient seine Autonomie nicht wahrnehmen will oder gar aus der Schwere seines Zustandes nicht mehr wahrnehmen kann, so muss auch nicht als primärer Reflex die Suche nach einer «Patientenverfügung» ausgelöst werden. Auch eine Patientenverfügung nützt wenig, wenn deren Inhalte nicht respektvoll behandelt werden.

\section{Ausblick: action and choice}

Unsere hochtechnisierte Medizin besteht aus schnell anwachsenden, komplexen, politisch und hierarchisch verschachtelten Handlungs- (action) und Wahl- (choice) Möglichkeiten [3]. Health Care Professionals müssen ermutigt werden, Handlungsketten als solche zu erkennen und diese auch moralisch zu reflektieren. Hier kann die klinische Ethik helfen. Ausserdem dürfen Patienten nicht mit Wahlmöglichkeiten alleingelassen werden, das wäre eine missverständliche Auslegung des Konzepts der Patientenautonomie. Auf solche Missverständnisse muss die klinische Ethik hinweisen.

\section{Danksagung}

Die Forschungsarbeit von R. Porz wird unterstützt durch die SAMW: «Zur Erweiterung der Methodikkompetenzen im Bereich der klinischen Ethik in der Schweiz» (KSZ-Fonds 2009-2010). Ein Dank an Christina aus der Au für die kritische Durchsicht des vorliegenden Textes.

\section{Literatur}

1 Vgl. Porz R. Auf dem Weg zu einer aufgeklärten angewandten Ethik: Hindernisse, Gefahren und Chancen. In: Bioethica Forum. 2009;2(2):100-2. Siehe auch Porz R, Laederach K. Ethikberatung im Spital - einige Überlegungen. In: SGBE Bulletin. 2010;60:4-6.

2 Vgl. Beauchamp TL, Childress JF. Principles of Biomedical Ethics. Oxford: Oxford University Press; 2009. Insbesondere: «Respect for autonomy (...) is a professional obligation (in health care). Autonomous choice is a right - not a duty - of patients.» (Betonung im Originaltext, S. 107).

3 Vgl. Rommetveit K. Action and Choice in Second Modernity. Bergen: Bergen University Press; 2006. 